\title{
Asunercept as an innovative therapeutic approach for recurrent glioblastoma and other malignancies
}

This article was published in the following Dove Press journal:

Cancer Management and Research

\author{
Andriy Krendyukov \\ Christian Gieffers \\ Apogenix AG, Heidelberg, Germany
}

Correspondence: Andriy Krendyukov Apogenix AG, Im Neuenheimer Feld 584, Heidelberg 69120, Germany

Email andriy.krendyukov@apogenix.com

\begin{abstract}
Glioblastoma is the most common and aggressive malignant tumor of the central nervous system. Despite the existing high unmet medical needs, the past few decades have seen no notable improvement in overall survival for glioblastoma patients. One active area of research to develop new therapeutic options for this disease is focusing on the CD95/Fas receptor and its ligand CD95L/FasL. It is now recognized that in addition to its role in programmed cell death, CD95/CD95L signaling is involved in a wide range of other apoptotic and non-apoptotic pathways directed toward T-effector cells and cells in the tumor microenvironment involved in tumor progression and invasiveness. Asunercept is a first-in-class recombinant glycosylated fusion protein, which has been designed to selectively bind to CD95L and therefore disrupt CD95/CD95L signaling. The current report provides a brief overview of the role of the CD95/CD95L signaling pathway in cancer pathogenesis and discusses how asunercept was designed to bind and neutralize CD95L and disrupt signaling thereby potentially improving outcomes in glioblastoma and other malignancies.
\end{abstract}

Keywords: CD95/CD95L, apoptosis, asunercept, APG101, glioblastoma, immuno-oncology

\section{Short report}

Glioblastoma (isocitrate dehydrogenase (IDH)-wildtype, IDH-mutant, and NOS glioblastoma according to the recent World Health Organization (WHO) classification of tumors of the central nervous system $)^{1}$ is the most common and aggressive malignant tumor of the central nervous system, with an age-adjusted incidence rate of 3.21 per $100,000 .^{2}$ Current guideline recommendations for patients with newly diagnosed glioblastoma consist of maximal surgical resection where safe and possible, and radiotherapy with concomitant and adjuvant temozolomide (TMZ) over several consecutive cycles. ${ }^{3-6}$ However, as glioblastoma is characterized by diffuse infiltrative growth and frequently found in eloquent brain areas, extensive surgical resection is not generally feasible. Infiltrating cancer cells invariably remain, leading to recurrence and disease progression with a relative survival for the majority of patients of 12-15 months under available therapy.

Recurrent glioblastoma has no well-established standard of care. Treatment options may include repeat surgery, re-irradiation, TMZ, PCV (procarbazine, CCNU [lomustine], and vincristine) or single-agent CCNU, and supportive care where it is needed. ${ }^{3,4,6}$ Access to ongoing investigational clinical trials is also considered a key therapeutic option, if available. However, many innovative therapeutics have failed to demonstrate clinical efficacy in newly diagnosed and recurrent glioblastoma, and in the last decade, only a few novel treatments have 
been approved. ${ }^{5}$ In patients with recurrent glioblastoma, prognosis is poor, on average only about 1 year from diagnosis, ${ }^{7,8}$ with 5 -year survival rates less than $5.6 \%{ }^{2}$ or even lower after disease progression.

Much research is ongoing to develop new therapeutic options for this disease. An approach targeting programmed cell death (apoptosis) may be one avenue to advance the treatment of recurrent glioblastoma, pending further confirmation in randomized, controlled trials. CD95/Fas and its ligand CD95L/FasL have been recognized for several decades for their role in apoptosis and as cancer hallmarks; however, only in recent years has clinical evidence emerged confirming CD95L as a promising novel target for the treatment of recurrent glioblastoma and potentially other malignancies. This short report describes the scientific rationale for CD95L inhibition in recurrent glioblastoma and presents evidence for asunercept, a selective CD95L inhibitor.

\section{Role of CD95/CD95L signaling pathway in cancer}

The CD95 (Fas or APO-1) receptor is a member of the tumor necrosis factor receptor superfamily and interaction with its natural ligand $(\mathrm{CD} 95 \mathrm{~L})$ has been implicated in several pathways of cancer pathogenesis. CD95 is a transmembrane protein with an extracellular ligand-binding domain and an intracellular signaling domain. In the classical case, extracellular binding of CD95L to CD95 triggers intracellular formation of the death-inducing signaling complex and induction of cellular apoptosis, including that of cancer cells. However, apoptotic signaling is frequently disrupted in cancers, and a number of lines of evidence suggest that the CD95/CD95L pathway plays a crucial role in the escape of tumor cells from immune surveillance and in the induction of cancer resistance to radiotherapy and immunotherapy. Inhibition of CD95/CD95L interaction might, therefore, represent a potential therapeutic approach for cancer immunotherapy. ${ }^{9-11}$

Concomitantly, depending on the tissue and the conditions, CD95/CD95L signaling can also mediate apoptotic and nonapoptotic signaling pathways directed toward T-effector cells (CD8+), including their differentiation and infiltration into the tumor microenvironment (TME), suggesting it may serve a role as an immune checkpoint. ${ }^{12}$ A number of other cells in the TME, including the tumor endothelium, and cancer-associated fibroblasts can also express CD95L and therefore induce T-cell apoptosis or suppress its function, for example when induced by angiogenic growth factors. ${ }^{10,11,13}$ Some evidence also suggests that the CD95/CD95L path might be connected to maintenance, and probably survival, of cancer stem cells (CSC), which can be associated with cancer cell persistence and relapse after radiation and/or chemotherapy, and lead to invasive growth. ${ }^{10} \mathrm{CD} 95$ is required for $\mathrm{CSC}$ survival and stimulation of $\mathrm{CD} 95$ on multiple tumor cells has been shown to result in conversion from non-CSC to CSC, independent of its apoptosis-inducing function, suggesting that CD95 expression could be a reliable surface marker for targeted therapy. ${ }^{14}$

\section{Scientific rationale for CD95L inhibition as a therapeutic approach in recurrent glioblastoma}

Increasing evidence shows that the non-apoptotic CD95/ CD95L signaling pathways play an important role in glioblastoma invasiveness and progression, which is characterized by infiltrative and rapid growth of tumor cells into the surrounding intact brain tissue. The binding of CD95L to the CD95 receptor on glioblastoma cells stimulates tyrosine kinases including the Yes member of the Src family and the p85 subunit of phosphatidylinositol 3 kinase (PI-3K), which along with increased expression of matrix metalloproteinase (MMP) have been described as underlying mechanisms of tumor invasion. ${ }^{15}$ When CD95 activity was neutralized, the invasive migration of glioblastoma cells was significantly reduced. ${ }^{15}$

It has been suggested that the recurrence-initiating cancer cells that emerge from the tumor cell population surviving initial therapy have stem cell-like properties because they can initiate a recurrent glioblastoma with a diversity of tumor cells. ${ }^{16}$ In addition, such cells are more aggressive than primary tumor-derived glioblastoma stem cells, ${ }^{17}$ which is consistent with the shorter survival of patients with recurrent glioblastoma; median overall survival after recurrence is 6.2 months despite treatment. ${ }^{18}$

Given the important role of CD95/CD95L signaling in tumor progression and recurrence, a number of lines of research are focusing on this pathway as a therapeutic target for recurrent glioblastoma. Asunercept is a first-in-class recombinant glycosylated fusion protein comprising the extracellular domain of human CD95 linked to the Fc domain of human IgG1. It has been designed to selectively bind to CD95L and therefore disrupt CD95/CD95L signaling. The scientific rationale for asunercept in recurrent glioblastoma is supported by a number of in vitro and in vivo non-clinical studies. CD95L is overexpressed in glioblastoma cells, which 
are largely resistant to CD95-mediated apoptosis resulting in their growth and invasiveness. Stimulation of glioblastoma cell lines (both apoptosis-resistant and apoptosis-sensitive) with CD95L increased migration of cells in the apoptosisresistant cell line, but not in the apoptosis-sensitive cell line, and could be prevented by blocking MMP activity. ${ }^{15}$ In murine glioblastoma cell lines, knockdown of the CD95L gene had no effect on overall cell viability nor the ability of the cells to form tumor spheroids, but it reduced tumor cell invasiveness, which could be restored by addition of soluble recombinant CD95L and inhibited by asunercept. ${ }^{19}$ Together these results suggest that $\mathrm{CD} 95 \mathrm{~L}$ from both autocrine and paracrine sources can contribute to the invasive nature of glioblastoma cells, and that asunercept can reduce this invasiveness by neutralizing CD95L.

In vivo models involving intracranial injection of cells from a murine glioblastoma into a syngeneic host may better reflect the TME and have illustrated the requirement for tumor/host interaction. Two weeks after intracranial injection there was significantly increased expression of both CD95 and CD95L in cells from the implanted tumor compared with levels expressed in cultured cells. ${ }^{15}$ Furthermore, when cells extracted from the implanted tumors were incubated with a CD95L neutralizing antibody, the invasive potential of the tumor cells was reduced by approximately 50\%. Using a similar syngeneic mouse model of glioblastoma, asunercept was associated with a dose-dependent inhibition of tumor growth when assessed by MRI volumetry. ${ }^{20}$

Non-clinical models also demonstrate an increase in invasive growth of glioblastoma cells following radiotherapy, similar to that which is observed in patients. However, asunercept treatment was able to counteract this radiotherapytriggered invasive potential of glioma-derived malignant cells and was associated with a survival advantage of up to 18 days over the control-arm $(p<0.05) .{ }^{20}$ Interestingly, in the same research, asunercept labeled with ${ }^{123} \mathrm{I}$ crossed the blood-brain barrier and enriched glioblastoma cells, suggesting a synergistic effect in combination with radiotherapy or chemotherapy. ${ }^{20}$

\section{Asunercept clinical evidence in recurrent glioblastoma patients}

The rationale for disruption of the CD95/CD95L pathway with asunercept and its clinical implications have been confirmed in a proof-of-concept Phase II study which evaluated the combination of asunercept and radiotherapy vs radiotherapy alone in patients with recurrent glioblastoma. ${ }^{21}$
Eligible patients were those experiencing recurrence of a histologically confirmed glioblastoma that was either not resectable or in whom there remained residual tumor after resection. Rates of progression-free survival (PFS) at 6 months were $3.8 \%$ (95\% CI $0.1-19.6)$ for radiotherapy alone and $20.7 \%$ (95\% CI 11.2-33.4) for the asunercept/ radiotherapy combination ( $p=0.048$ ). Median PFS was 2.5 months (95\% CI 2.3-3.8 months) and 4.5 months (95\% CI 3.7-5.4 months), respectively, with a hazard ratio of 0.49 ( $p=0.0162$ ). In this trial, the combination of asunercept and radiotherapy was well tolerated with no serious adverse events and no impairment of radiotherapy tolerability. A recent update on overall survival of patients has revealed that $7 \%$ of asunercept/radiotherapy-treated recurrent glioblastoma patients were alive after 5 years compared to $0 \%$ of the patients treated with radiotherapy alone. ${ }^{22}$ The combination was associated with a significant improvement in time to first deterioration and maintained quality of life compared with radiotherapy treatment alone. ${ }^{23}$

In an attempt to identify biomarkers that predict the treatment response of asunercept, archived primary tumor tissues from patients with glioblastoma at first or second progression were analyzed for expression levels of CD95L and DNA methylation status of the CD95L promoter. ${ }^{21,24}$ These studies suggested that patients displaying a low level of methylation responded best to asunercept therapy (hazard ratio $0.19,95 \%$ CI 0.06-0.58). There was a significant survival benefit for patients with low methylation when treated with a combination of asunercept and radiotherapy vs radiotherapy alone (HR 0.34, $p=0.024$ ), suggesting differential CD95L promoter methylation may represent a potential biomarker to predict response to asunercept treatment. Preliminary results suggest that CD95L promoter methylation correlates with the respective transcriptional activity and might, therefore, be responsible for regulating CD95L protein levels. Further research is warranted to determine whether this potential biomarker can also predict which patients would respond best to a combination of asunercept with radiotherapy or with chemotherapy.

\section{Asunercept clinical evidence in myelodysplastic syndrome (MDS) patients}

The CD95/CD95L signaling pathway and asunercept, as a selective CD95L inhibitor, offer broad potential applicability to a range of malignancies with aberrant CD95/CD95L signaling, beyond recurrent glioblastoma patients. In MDS, a decrease in physiological erythropoiesis is associated with 
increased apoptosis of $\mathrm{CD} 34+$ hematopoietic progenitor cells. CD95 is overexpressed on $\mathrm{CD} 34+$ progenitors and erythrocytes in around two thirds of patients with lower risk MDS, and its activation is thought to negatively regulate erythrocyte production in the bone marrow. ${ }^{25}$ Hypothesizing that asunercept inhibition of CD95L signaling would protect the CD34+ erythrocyte precursors from CD95L-induced apoptosis, a proof-of-concept study was conducted in 20 transfusion-dependent low- and intermediate-risk MDS patients resistant to treatment with erythropoiesis-stimulating agents. Asunercept administration was associated with a decrease in transfusion need and frequency and was well tolerated. ${ }^{26}$ Analysis of bone marrow biopsies from these patients revealed that the decreased transfusion need during the study was associated with an increase in burst-forming unit-erythroid progenitor activity compared to baseline levels. ${ }^{26}$ These results confirm that direct inhibition of CD95/CD95L signaling plays an important role in rescuing erythropoiesis with potential clinical implications for hematological malignancies such as MDS.

\section{Asunercept safety and tolerability}

The safety and tolerability of asunercept have been evaluated in healthy volunteers as well as in proof-of-concept clinical studies in patients with recurrent glioblastoma and transfusion-dependent patients with low- and intermediate-risk MDS. $^{21,26,27}$ The proportions of subjects reporting any adverse event after administration of asunercept or placebo were similar at $60.0 \%$ and $57.1 \%$, respectively. ${ }^{27}$ Only one adverse event in an asunercept-treated subject, one episode of mild headache, was considered related to the investigational medicine. In patients with recurrent glioblastoma randomized to asunercept $400 \mathrm{mg}$ /week plus radiotherapy or radiotherapy alone, the combination was well tolerated and not associated with any serious adverse events. ${ }^{21}$ Mild and moderate nervous system disorders, mostly headache, were more common in the combination group $(20.7 \%$ and $53.4 \%$, respectively) than in patients receiving radiotherapy alone (15.4\% and $30.8 \%$, respectively). Asunercept was tested in a clinical study with low- and intermediate-risk MDS patients who received doses of $100 \mathrm{mg}$ or $400 \mathrm{mg} /$ week. The relationship of adverse events to investigational medication was suspected with a possible or probable categories in $9(45 \%)$ patients. ${ }^{26}$ Overall two patients experienced serious adverse events that were suspected to be drug-related, one patient experienced a hypersensitivity reaction and one patient with pre-existing neutropenia had septic complications.

\section{Potential implications in other tumors}

As research with the selective CD95L inhibitor asunercept advances, opportunities might be directed toward other tumor types with high levels of CD95L expression on cancerand TME cells. Non-clinical studies conducted using the TiRP mouse melanoma model, which mimics resistance signature and TME observed in human melanomas, have shown that these tumors are enriched in myeloid-derived suppressor cells which express high levels of CD95L and subsequently induce apoptosis of tumor-infiltrating lymphocytes. ${ }^{9}$ Importantly, this could be prevented by inhibiting CD95L, providing a rationale for the use of asunercept beyond recurrent glioblastoma, for example in cutaneous melanoma. It is reasonable to consider that the use of asunercept may be particularly effective in combination with other immune checkpoint therapies antagonizing T-lymphocyte-associated protein 4 (CTLA-4) and programmed cell death protein 1 (PD-1). ${ }^{28}$ Analysis of human cancer tissue samples has also found high levels of CD95L expression associated with certain types of breast, colon, renal, bladder, prostate, head and neck, pancreatic, and ovarian adenocarcinomas, ${ }^{11}$ suggesting that targeting the CD95/CD95L pathway with asunercept may also offer a promising innovative treatment approach and should be considered for further evaluation and confirmation in clinical studies.

\section{Conclusion}

Selective inhibition of the CD95/CD95L pathway plays a critical role in reducing the invasive growth that characterizes recurrent glioblastoma and potentially other tumors with high levels of CD95L expression, and in parallel serves to improve the ability of cytotoxic $\mathrm{T}$ cells to infiltrate tumors. Proof-of-concept studies have demonstrated that asunercept,a fully human selective CD95L inhibitor, might provide clinical efficacy in patients with recurrent glioblastoma and low to intermediate-risk MDS with a good tolerability profile at intended therapeutic dose. Targeting the CD95/CD95L pathway with a tailored therapy such as asunercept may, therefore, offer a promising innovative treatment alternative and is currently under further clinical evaluation.

\section{Disclosure}

AK and CG are full time employees of Apogenix AG. AK was a former Hexal AG employee within the last 36 
months. The authors report no other conflicts of interest in this work.

\section{References}

1. Louis DN, Perry A, Reifenberger G, et al. The 2016 World Health Organization classification of tumors of the central nervous system: a summary. Acta Neuropathol. 2016;131(6):803-820. doi:10.1007/ s00401-016-1545-1

2. Ostrom QT, Gittleman H, Truitt G, et al. CBTRUS statistical report: primary brain and other central nervous system tumors diagnosed in the United States in 2011-2015. Neuro Oncol. 2018;20(suppl_4):iv1iv86. doi:10.1093/neuonc/noy131

3. Stupp R, Brada M, van Den Bent MJ, Tonn J-C, Pentheroudakis G. ESMO guidelines working group. high-grade glioma: ESMO clinical practice guidelines for diagnosis, treatment and follow-up. Ann Oncol. 2014;25(Suppl3):iii93-iii101. doi:10.1093/annonc/mdu050

4. Weller M, van Den Bent M, Hopkins K, et al. European Association for Neuro-Oncology (EANO) task force on malignant glioma. EANO guideline for the diagnosis and treatment of anaplastic gliomas and glioblastoma. Lancet Oncol. 2014;15(9):e395-e403. doi:10.1016/ S1470-2045(14)70011-7

5. National Comprehensive Cancer Network. NCCN clinical practice guidelines in oncology. Version 1.2018. Central Nervous System Cancers. Available from: https://www.nccn.org/. Accessed July 31, 2019.

6. National Institute for Health and Care Excellence. Brain Tumours (primary) and Brain Metastases in Adults. NICE guideline (NG99); 2018. Available from: https://www.nice.org.uk/guidance/ng99. Accessed July 31, 2019.

7. Stupp R, Hegi ME, Mason WP, et al. Effects of radiotherapy with concomitant and adjuvant temozolomide versus radiotherapy alone on survival in glioblastoma in a randomised phase III study: 5-year analysis of the EORTC-NCIC trial. Lancet Oncol. 2009;10:459-466. doi:10.1016/S1470-2045(09)70234-7

8. Wick W, Osswald M, Wick A, Winkler F. Treatment of glioblastoma in adults. Ther Adv Neurol Disord. 2018;11:1756286418790452. doi:10.1177/1756286418790452

9. Zhu J, Powis de Tenbossche CG, Cané S, et al. Resistance to cancer immunotherapy mediated by apoptosis of tumor-infiltrating lymphocytes. Nat Commun. 2017;8(1):1404. doi:10.1038/s41467-017-00784-1

10. Peter ME, Hadji A, Murmann AE, et al. The role of CD95 and CD95 ligand in cancer. Cell Death Differ. 2015;22(4):549-559. doi:10.1038/ cdd. 2015.3

11. Motz GT, Santoro SP, Wang LP, et al. Tumor endothelium fasL establishes a selective immune barrier promoting tolerance in tumors. Nat Med. 2014;20(6):607-615. doi:10.1038/nm.3541

12. Yi F, Frazzette N, Cruz AC, Klebanoff CA, Siegel RM. Beyond cell death: new functions for TNF family cytokines in autoimmunity and tumor immunotherapy. Trends Mol Med. 2018;24(7):642-653. doi:10.1016/j.molmed.2018.05.004

13. Lakins MA, Ghorani E, Munir H, Martins CP, Shields JD. Cancerassociated fibroblasts induce antigen-specific deletion of $\mathrm{CD}^{+} \mathrm{T}$ cells to protect tumour cells. Nat Commun. 2018;9(1):948. doi:10.1038/s41467-018-03347-0

14. Ceppi P, Hadji A, Kohlhapp FJ, et al. CD95 and CD95L promote and protect cancer stem cells. Nat Commun. 2014;5:5238. doi:10.1038/ ncomms5972
15. Kleber S, Sancho-Martinez I, Wiestler B, et al. Yes and PI3K bind CD95 to signal invasion of glioblastoma. Cancer Cell. 2008;13 (3):235-248. doi:10.1016/j.ccr.2008.02.003

16. Osuka S, Van Meir EG. Overcoming therapeutic resistance in glioblastoma: the way forward. J Clin Invest. 2017;127(2):415-426. doi:10.1172/JCI89587

17. Huang Q, Zhang QB, Dong J, et al. Glioma stem cells are more aggressive in recurrent tumors with malignant progression than in the primary tumor, and both can be maintained long-term in vitro. $B M C$ Cancer. 2008;8:304. doi:10.1186/1471-2407-8-172

18. Gorlia T, Stupp R, Brandes AA, et al. New prognostic factors and calculators for outcome prediction in patients with recurrent glioblastoma: a pooled analysis of EORTC brain tumour group phase I and II clinical trials. Eur J Cancer. 2012;48:1176-1184. doi:10.1016/j. ejca.2012.02.004

19. Merz C, Strecker A, Sykora J, et al. Neutralization of the CD95 ligand by APG101 inhibits invasion of glioma cells in vitro. Anticancer Drugs. 2015;26(7):716-727. doi:10.1097/CAD.0000000000000237

20. Blaes J, Thomé CM, Pfenning PN, et al. Inhibition of CD95/CD95L (FAS/FASLG) signalling with APG101 prevents invasion and enhances radiation therapy for glioblastoma. Mol Cancer Res. 2018;16(5):767-776. doi:10.1158/1541-7786.MCR-17-0563

21. Wick W, Fricke H, Junge K, et al. A phase II, randomized, study of weekly APG101+reirradiation versus reirradiation in progressive glioblastoma. Clin Cancer Res. 2014;20:6304-6313. doi:10.1158/ 1078-0432.CCR-13-3045

22. Fricke H, Hanke N, Kunz C, et al. Asunercept plus radiotherapy in relapsed glioblastoma. Update on five years overall survival of study NCT01071837 and development of a population-PK-tumor growth inhibition survival model. Poster presented at 23 annual meeting of society for neuro oncology, New Orleans, Louisiana 15-18 Nov 2018. Neuro-Oncology. 2018;20(Suppl 6):vi3.

23. Wick W, Krendyukov A, Junge K, et al. Longitudinal Analysis of Quality of Life following Treatment with Asunercept Plus Reirradiation versus Reirradiation in Progressive Glioblastoma Patients. J Clin Oncol. 2019;37(15 Suppl):Abstract 2022.

24. Gieffers C, Kunz C, Sykora J, et al. Methylation of a single CpG site in the CD95-ligand promoter is a biomarker predicting the response to therapy with APG101 in glioblastoma. [abstract]. In: proceedings of the 107th annual meeting of the American association for cancer research. 2016 Apr 16-20; New Orleans, LA. Philadelphia (PA): AACR. Cancer Res. 2016;76(14Suppl):Abstractnr 464. doi:10.1158/ 0008-5472.CAN-16-0584

25. Raimbault A, Pierre-Eugene C, Rouquette A, et al. Groupe francophone des myélodysplasies. APG101 efficiently rescues erythropoiesis in lower risk myelodysplastic syndromes with severe impairment of hematopoiesis. Oncotarget. 2016;7(12):14898-14911. doi:10.18632/oncotarget.7469

26. Boch T, Luft T, Metzgeroth G, et al. Safety and efficacy of the CD95ligand inhibitor asunercept in transfusion-dependent patients with low and intermediate risk MDS. Leuk Res. 2018;68:62-69. doi:10.1016/j.leukres.2018.03.007

27. Tuettenberg J, Seiz M, Debatin KM, et al. Pharmacokinetics, pharmacodynamics, safety and tolerability of APG101, a CD95-Fc fusion protein, in healthy volunteers and two glioma patients. Int Immunopharmacol. 2012;13(1):93-100. doi:10.1016/j.intimp.2012.03.004

28. Seidel JA, Otsuka A, Kabashima K. Anti-PD-1 and anti-CTLA-4 therapies in cancer: mechanisms of action, efficacy, and limitations. Front Oncol. 2018;8:86. doi:10.3389/fonc.2018.00086 


\section{Publish your work in this journal}

Cancer Management and Research is an international, peer-reviewed open access journal focusing on cancer research and the optimal use of preventative and integrated treatment interventions to achieve improved outcomes, enhanced survival and quality of life for the cancer patient.
The manuscript management system is completely online and includes a very quick and fair peer-review system, which is all easy to use. Visit http://www.dovepress.com/testimonials.php to read real quotes from published authors. 